Fetal Diagnosis and Therapy
Fetal Diagn Ther 2016;39:1-3

DOI: $10.1159 / 000441452$
Received: August 5, 2015

Accepted after revision: September 23, 2015

Published online: November 7, 2015

\title{
The Microbiota and Transgenomic Networks: Potential Implications for Maternal-Fetal Medicine
}

\author{
Joaquin Santolaya-Forgas ${ }^{a}$ Ryan Townsend ${ }^{b}$ Jacobo L. Santolaya ${ }^{b}$ \\ Priya Patel $^{b}$ Guadalupe Herrera-Garcia $^{b}$ V. Daniel Castracane ${ }^{c}$ \\ ${ }^{a}$ The Perinatal Institute, Jersey Shore University Medical Center, Neptune, N.J., b Department of Obstetrics and \\ Gynecology, Rutgers-Robert Wood Johnson Medical School, New Brunswick, N.J., and 'Texas Tech University Health \\ Science Center, Odessa, Tex., USA
}

\section{Key Words}

Microbiota · Microbiotal stimulus · Coevolution · Biological adaptation - Transcription factors · Microbial invasion of the amniotic cavity

\begin{abstract}
The maternal microbiota has long been considered a potential cause for adverse perinatal outcomes. Gene expression regulators in prokaryotic and eukaryotic cells are influenced by changes in their microenvironments. We propose the novel idea that during in utero development, an adaptive and dynamic gene-regulatory cross talk might exist between the host genome and the maternal microbiota. Understanding these cross talks could increase the appreciation for the discovery of new diagnostics and therapeutics in maternalfetal medicine.

๑) 2015 S. Karger AG, Basel
\end{abstract}

Modern medicine recognizes the importance of synergistic relationships between different genomes and biological systems. The bacterial types contained within distinct body niches reflect the evolution of the mutualistic relationship between human beings and the impressive biodiversity of colonizing bacteria (human microbiota). It has been proposed that evolutionary adaptations to microbial epidemics $[1,2]$ and the responses to newly acquired microbes at birth affect the biodiversity of bacteria contained in the neonate's body $[3,4]$ and in the mature individual $[5,6]$. However, new anatomical sites for the microbiota including the placenta have been discovered, changing the interpretation of how the fetus becomes colonized. Antibiotic therapy during the perinatal period and poor quality or quantity of breast feeding also contribute to the intestinal dysbiosis observed in patients with necrotizing enterocolitis, inflammatory bowel disease, autism, obesity and colon cancer [7-13]. All of these observations taken together with the completion of the human microbiome project have elevated the interest in this field of medicine to an even higher level [14]. Moreover, antimicrobials that protect the beneficial effect of the commensal microbiome and prevent the devastating effects of multidrug-resistant pathogens are sought after [15].

Without doubt, validation of genotype-phenotype associations is playing a critical role in the understanding of how cells acquire the mature phenotype $[16,17]$. However, we propose that the adaptive responses of the host genome to the microbiota during distinct developmental

\section{KARGER}

E-Mail karger@karger.com

www.karger.com/fdt
(C) 2015 S. Karger AG, Basel

$1015-3837 / 15 / 0391-0001 \$ 39.50 / 0$
Joaquin Santolaya-Forgas, MD, $\mathrm{PhD}$

Department of Genetics, Rutgers-The State University of New Jersey Clinical Genetics and Genomics, Meridian Health System

Medical Arts Building, Suite 204, 1944 Route 33, Neptune, NJ 07753 (USA)

E-Mail jsantolaya@ meridianhealth.com 
windows could also contribute to the mature phenotype of human cells [5]. Thus, the transcription control imposed upon genes by bacterial extracellular signals could play a major role in human cellular gene expression. Deciphering if synergistic relationships among the maternal, fetal and microbiome gene-regulatory mechanisms exist could provide novel mechanistic information concerning the shape and function of the developing placenta and fetus $[5,18]$.

Currently we do not know if a sterile intra-amniotic environment is a requirement for a normal intrauterine development $[19,20]$. We do know that over the course of pregnancy, there are progressive maternal hemodynamic and body weight changes, a functional resistance to insulin and a pro-inflammatory setting, which together favor variations in the maternal microbiome load and composition. Therefore, a selective bacterial colonization of the placenta and the fetus might exist [21, 22]. If a commensal intra-amniotic and fetal microbiota is confirmed using genome-wide association studies along with functional genomics and metagenomic approaches, then studies aimed at (1) scrutinizing how the lining of the female reproductive tract functions as a selective barrier to the microbiota, (2) the mechanisms used by bacteria to cross intact chorioamniotic membranes in normal and pathological states and (3) the potential role of intra-amniotic bacteria in fetal metabolism and immune regulation will follow [23-26].

The morphological and developmental evolution across species in many ways reflects the adaptation to the environment. It is now important that we investigate if transgenomic networks between the microbiota and the human genome exist and if the host genome can respond to the changing microenvironment created by the microbiota. Although we still have very limited knowledge of the evolution of the gene-regulatory machinery in bacteria and higher organisms, there is evidence suggesting that the cell phenotype and function adapts to the environment through temporal-spatial regulation of gene expression [27]. Mechanistically, this adaptation has occurred through the specialization of conserved motifs that allow for the gain or loss of transcription factors (TF) binding site(s) for selective gene expression. In other words, changes in DNA architecture that affect the promoter, the position of the gene or the configuration of the binding site for the TF and the transcriptional machinery, stand behind the differential regulation of the timing of gene expression [28]. There is also evidence suggesting that the gene-regulatory machinery in bacteria is made of hubs of co-regulatory networks, autonomous hubs to reg- ulate a diverse array of genes in response to DNA damage or starvation, operons and regulons. Eukaryotes on the other hand use regulatory systems that are based on associations between different TFs that bring about co-expression of genes scattered around the chromosome. Overall, TFs are activators, repressors or dual regulators. TFs can also be classified as internal sensing, external sensing, hybrid class, chromosomal proteins associated with DNA-curvature status and TFs in two-component signal transduction systems [29]. If we focus on the action of TFs on the gene expression-regulatory machinery, we can anticipate responses to environmental changes through circuits consisting of TF/DNA-binding proteins that target gene promoters/repressors. Rewiring of these direct one-component transcription systems can explain many of the observed pleiotropic cellular responses. Other responses observed in higher-order organisms must depend on multistage circuits that have feedback loops, feedforward loops, regulatory cascades, two-component systems and connector proteins. These higher-level regulatory circuits can also be classified as TF repressors (multiple-input motifs), TF activators (feedforward motifs) and TF of neither tendency (single-input motif) [30]. Since a TF and a sensory element are separately needed in both bacteria and eukaryotes, the possibility of an even higher-level regulatory system that allows for the sharing of components of the machinery of gene expression regulators between the changing microbiota, maternal cells and fetal cells might exist. In this purely hypothetical scheme, combinatory regulation could be part of the biological complexity via a relative number of regulatory elements during in utero development. If this is confirmed, then bacteria, maternal cells and fetal cells are interacting functional units: bacteria through this higher-order regulatory network could differentially boost distinct genetic makeups of the mother and fetus, contributing to the shaping of human kind. With future studies, the potential for predicting congenital disorders associated with the patient's distinct microbiota is immense. Although only speculation at this point, maternal microbiomes could be used as a means of detecting likelihoods of perinatal complications or as biomarkers for in utero diagnostics and therapies.

In summary, for several decades, the human microbiota has been the subject of intense interest. Until recently, it was generally accepted that human intrauterine development takes place in a sterile environment and that infants delivered by a cesarean operation are colonized by maternal skin surface bacteria, while infants delivered naturally are colonized by bacteria from the mother's va- 
gina. We are now becoming aware that the intra-amniotic cavity might not be sterile and that bacteria, the mother and the fetus could be more than interacting functional units. Indeed, a dynamic regulatory cross talk between the host genome and the microbiome through common gene expression regulators might exist. If this hypothesis is true, then we could forecast two important implications. First, this higher-order transcription network contributes to the dynamic reconfiguration of human cells and bacteria. Second, the maternal microbiota could be more than a spectator of intrauterine development and differentially boost the genetic makeup of the mother and fetus. Future studies on this interplay between the microbiome and the maternal and fetal genomes could provide a better understanding of fetal development as well as novel in utero treatments to reduce the risk for neonatal dysbiosis linked with necrotizing enterocolitis, inflammatory bowel disease, autism, obesity and cancer.

\section{References}

-1 Li LM, Grassly NC, Fraser C: Genomic analysis of emerging pathogens: methods, application and future trends Genome Biol 2014;15: 541-549.

-2 Turnbaugh PJ, Ley RE, Hamady M, FraserLiggett C, Knight R, Gordon JI: The human microbiome project: exploring the microbial part of ourselves in a changing world. Nature 2007;449:804-810.

$\checkmark 3$ Payne MS, Bayatibojakhi S: Exploring preterm birth as a polymicrobial disease: an overview of the uterine microbiome. Front Immunol 2014;5:595.

$\checkmark 4$ Dominguez-Bello MG, Costello EK, Contreras M, Magris M, Hidalgo G, Fierer N, et al: Delivery mode shapes the acquisition and structure of the initial microbiota across multiple body habitats in newborns. Proc Natl Acad Sci USA 2010;107:11971-11975.

$>5$ Gohir W Ratcliffe EM, Sloboda D: Of the bugs that shape us: maternal obesity, the gut microbiome, and long-term disease risk. Pediatr Res 2014;77:196-204.

$\checkmark 6$ Leslie JL, Young VB: The rest of the story: the microbiome and gastrointestinal infections. Curr Opin Microbiol 2015;23:121-125.

$>7$ Quercia S, Candela M, Turroni S, Luiselli D, Rampelli S, et al: From lifetime to evolution: timescales of human gut microbiota adaptation. Front Microbiol 2014;5:587.

$>8$ LaTuga MS, Stuebe A, Seed PC: A review of the source and function of microbiota in breast milk. Semin Reprod Med 2014;32:6873.

$>9$ Miniello VL, Colosanto A, Cristofori F, Diaferio L, et al: Gut microbiota biomodulators, when the stork comes by the scalpel. Clin Chim Acta 2015, DOI: 10.1016/j.cca.2015. 01.022 .
10 Arumugam M, Raes J, Pelletier E, Le Paslier D, Yamada T, Mende DR, et al: Enterotypes of the human gut microbiome. Nature 2011; 473:174-180.

11 Grossi E, Terruzi V: The role of intestinal dysbiosis in the pathogenesis of autism: minireview. Int J Microbiol Adv Immunol 2014;2: 201.

-12 Russo AJ: Decreased plasma myeloperoxidase associated with probiotic therapy in autistic children. Clin Med Insights Pediatr 2015;9: 13-17.

13 Cox LM, Blaser MJ: Antibiotics in early life and obesity. Nat Rev Endocrinol 2015;11: 182-190.

14 Prince AL, Chu DM, Seferovic MD, Antony KM, Ma J, Aagard KM: The perinatal microbiome and pregnancy: moving beyond the vaginal microbiome. Cold Spring Harb Perspect Med 2015, DOI: 10.1101/cshperspect. a023051.

15 Beisel CL, Gomaa AA, Rarrongou R: A CRISPR design for next-generation antimicrobials. Genome Biol 2014;15:516.

16 Cho MK: Translating genomics into the clinic: moving to the post-Mendelian world. Genome Med 2009;1:7.

17 Simpson JT: Genome informatics 2014. Genome Biol 2014;15:543.

18 Gluckman PD, Hanson MA: Developmental plasticity and human disease research directions. J Intern Med 2007;261:461-471.

19 Rautava S, Luoto R, Salminen S, Isolauri E: Microbial contact during pregnancy, intestinal colonization and human disease. Nat Rev Gastroenterol Hepatol 2012;9:565-576.

20 Hanage WP: Microbiology: microbiome science needs a healthy dose of scepticism. Nature 2001;512:247-248.

-21 Amarasekara R, Jayasekara RW, Senanayake $\mathrm{H}$, Dissanayake VH: Microbiome of the placenta in pre-eclampsia supports the role of bacteria in the multifactorial cause of pre-eclampsia. J Obstet Gynaecol Res 2015;41:662669.
22 Koren O, Goodrich JK, Cullender TC, Spor A, Laitinen K, Bäckhed HK, et al: Host remodeling of the gut microbiome and metabolic changes during pregnancy. Cell 2012;150: 470-480.

23 Yarbrough VL, Winkle S, Herbst-Kralovetz MM: Antimicrobial peptides in female reproductive tract: a critical component of mucosal immune barrier with physiological and clinical implications. Hum Reprod Update 2015; 21:353-377.

24 Read TD, Massey R: Characterizing the genetic basis of bacterial phenotypes using genome-wide association studies: a new direction for bacteriology. Genome Med 2014;6: $1-11$.

25 Yoon SS, Kim K, Lee W-J: Functional genomic and metagenomics approaches to understanding gut microbiota-animal mutualism. Curr Opin Microbiol 2015;24:38-46.

26 Yang Y, Hu M, Zeng X, Liu X: Mass spectrometry-based proteomic approaches to study pathogenic bacteria-host interactions. Protein Cell 2015;6:265-274.

27 Cao J, Zhengyu L, Quingu C, Qianian X, Zhang Y, et al: Three-dimensional regulation of transcription. Protein Cell 2015;6:241-253.

28 Perez JC, Groisman E: Evolution of transcriptional regulatory circuits in bacteria. Cell 2009; 138:233-244.

29 Balaji S, Babu MM, Aravind L: Interplay between network structures, regulatory modes and sensing mechanisms of transcription factors in the transcriptional regulatory network of E. coli. J Mol Biol 2007;372:1108-1122.

-30 Babu MM, Luscombe NM, Aravind L, Gerstein M, Teichmann SA: Structure and evolution of transcriptional regulatory networks. Curr Opin Struct Biol 2004;14:283-291.
The Microbiota and Transgenomic Networks
Fetal Diagn Ther 2016;39:1-3 DOI: $10.1159 / 000441452$ 